\title{
Erratum to: Stimulated and Unstimulated Saliva Levels of Calcium and Magnesium in Giardiasis
}

\author{
Minoo Shaddel $^{1}$ - Iraj Mirzaii-Dizgah ${ }^{2} \cdot$ Khojasteh Sharifi-Sarasiabi $^{3} \cdot$ Zahra Kamali $^{3}$. \\ Mani Dastgheib ${ }^{4}$
}

Published online: 27 February 2017

(C) Springer Science+Business Media New York 2017

Erratum to: Biol Trace Elem Res (2017)

DOI 10.1007/s12011-017-0943-0

The original version of this article contained two errors: the author name Iraj Mirzaii-Dizgah was incorrectly written as Iraj Mirzaii Dizgah and the affiliation of Khojasteh SharifiSarasiabi was incorrectly written as Tropical and Infectious Research Center, Faculty of Medicine, Hormozgan University of Medical Sciences and Health Services, Bandar Abbas, Iran. It should have been Infectious and Tropical Diseases Research Center, Hormozgan Health Institute, Hormozgan University of Medical Sciences, Bandar Abbas, Iran.

The original article has been corrected.

The online version of the original article can be found at http://dx.doi. org/10.1007/s12011-017-0943-0.

Iraj Mirzaii-Dizgah

emirzaii@alumnus.tums.ac.ir

1 Department of Parasitology, Faculty of Medicine, Aja University of Medical Sciences, Tehran, Iran

2 Department of Physiology, Faculty of Medicine, Aja University of Medical Sciences, Tehran, Iran

3 Infectious and Tropical Diseases Research Center, Hormozgan Health Institute, Hormozgan University of Medical Sciences, Bandar Abbas, Iran

4 Taleghani Hospital, Abadan, Iran 